\title{
On the Maximum Number of Edges in Topological Graphs with no Four Pairwise Crossing Edges
}

\author{
Eyal Ackerman
}

Received: 21 August 2006 / Revised: 5 December 2007 / Accepted: 5 December 2007 /

Published online: 13 February 2009

(C) Springer Science+Business Media, LLC 2009

\begin{abstract}
A topological graph is called $k$-quasi-planar if it does not contain $k$ pairwise crossing edges. It is conjectured that for every fixed $k$, the maximum number of edges in a $k$-quasi-planar graph on $n$ vertices is $O(n)$. We provide an affirmative answer to the case $k=4$.
\end{abstract}

Keywords Geometric graphs · Topological graphs · Discharging method · Quasi-planar graphs

\section{Introduction}

A topological graph is a graph drawn in the plane with its vertices as points and its edges as Jordan arcs that connect corresponding points and do not contain any other vertex as an interior point. Throughout this paper we use the term arc when referring to an edge of a topological graph. We only consider graphs that do not contain loops or parallel arcs. We also assume that any two arcs of a topological graph have a finite number of intersection points that are either endpoints or crossing points. A planar graph is a graph that can be drawn in the plane without any pair of crossing arcs. One possible generalization of the notion of planar graphs are $k$-quasi-planar graphs. A $k$-quasi-planar graph is a topological graph with no $k$ pairwise crossing arcs. We denote by $f_{k}(n)$ the maximum number of arcs in such a graph on $n$ vertices.

Since 2-quasi-planar graphs are planar graphs, it follows from Euler's Polyhedral Formula that $f_{2}(n) \leq 3 n-6$. It is a well-known conjecture [3, Problem 3.3] that for any fixed $k$, there is a constant $C_{k}$ such that $f_{k}(n) \leq C_{k} n$. Agarwal et al. [2] were the first to prove this conjecture for $k=3$. Later, Pach et al. [4] simplified their proof and showed that $f_{3}(n) \leq 65 n$. Recently, Ackerman and Tardos [1] proved that $7 n-$

E. Ackerman $(\bowtie)$

Computer Science Dept., Technion-Israel Institute of Technology, Haifa 32000, Israel

e-mail: ackerman@cs.technion.ac.il 
$O(1) \leq f_{3}(n) \leq 8 n-\Omega(1)$ and provided a tight bound of $6.5 n-\Omega(1)$ for simple topological graphs (graphs in which every pair of arcs intersect at most once). For a fixed $k \geq 4$, the best upper bound for topological graphs is $O\left(n \log ^{4 k-12} n\right)$ [4], while for topological graphs with $x$-monotone arcs, Valtr [5] showed an upper bound of $O(n \log n)$. In this paper we extend the work of Ackerman and Tardos [1] to provide the first proof that $f_{4}(n)=O(n)$.

\section{Proof of the Main Theorem}

Since the (underlying abstract) graphs we consider contain no loops or parallel arcs, we have $f_{4}(1)=0$ and $f_{4}(2)=1$. For greater values of $n$, we prove the following theorem.

Theorem 1 For any integer $n>2$, every topological graph on $n$ vertices with no four pairwise crossing arcs has at most $72(n-2)$ arcs.

Proof It is easy to see that $f_{4}(3)=3<72(3-2)$. Let $G$ be a 4-quasi-planar graph on $n>3$ vertices. We denote by $V(G)$ the vertex set of $G$, and by $E(G)$ the arc set of $G$. Given an arc $e \in E(G)$ and two points $p$ and $q$ on $e$, we use the notation $\left.e\right|_{p, q}$ to denote the segment of $e$ between $p$ and $q$. For a vertex $v$, we denote by $d(v)$ the degree of $v$. If there is a vertex $v \in V(G)$ such that $d(v)<3$, then we can conclude the theorem by induction. Hence, we assume the that degree of every vertex in $G$ is at least three. Assume, w.l.o.g., that $G$ is drawn with the least possible number of crossings such that there are no four pairwise crossing arcs and that there are no three arcs crossing at the same point. Let $a_{1}$ and $a_{2}$ be two arcs of $G$ that intersect at least twice. A region bounded by segments of $a_{1}$ and $a_{2}$ that connect two consecutive intersection points is called a lens. We observe, as in [4], that $G$ has no empty lenses, that is, lenses that do not contain a vertex of $G$. If there were empty lenses, then the number of crossings in $G$ could be reduced. For the same reason, $G$ does not contain self-intersecting arcs.

Let $G^{\prime}$ be the (drawing of the) planar graph induced by $G$. That is, $V\left(G^{\prime}\right)=$ $V(G) \cup X(G)$, where $X(G)$ is the set of crossing points in $G$; and $e^{\prime} \in E\left(G^{\prime}\right)$ if $e^{\prime}$ is a segment of an arc of $G$ that connects two vertices in $V\left(G^{\prime}\right)$ and contains no other vertex from $V\left(G^{\prime}\right)$. Henceforth, the term edge refers to an edge of $G^{\prime}$, while the term arc refers to an edge of $G$. Denote by $F\left(G^{\prime}\right)$ the set of faces of $G^{\prime}$, and let $|f|$ be the number of edges along the boundary of a face $f \in F\left(G^{\prime}\right) .{ }^{1}$ Given a face $f$, we denote by $v(f)$ the number of vertices from $V(G)$ along the boundary of $f$ (we call these vertices original vertices). We will use the terms triangles, quadrilaterals, pentagons, and hexagons to refer to faces of size 3, 4, 5, and 6, respectively. An integer $m$ before the name of a face, denotes the number of original vertices on its boundary. For example, a 2-pentagon is a face of size 5 that has 2 original vertices on its boundary.

Following [1], we use the discharging method to prove the theorem. We begin by assigning a certain amount of charge to every face of $G^{\prime}$ such that the overall

\footnotetext{
${ }^{1}$ Note that an edge can sometimes appear twice along the boundary of a face.
} 


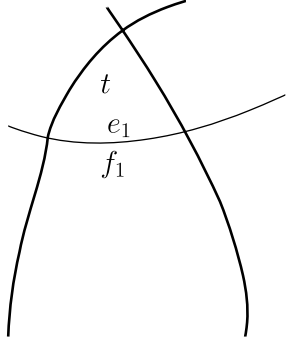

(a)

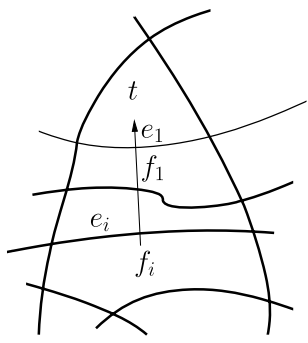

(b)

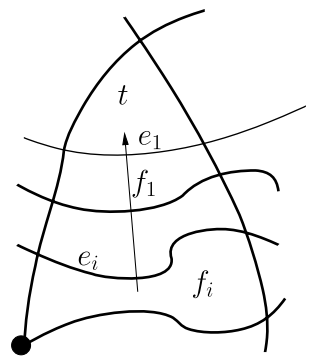

(c)

Fig. 1 Charging a 0-triangle

assigned charge is $O(n)$. Then, we redistribute the charges (discharging phase) such that every face has a nonnegative charge and the charge of every original vertex $v$ is proportional to $d(v)$. This implies that the overall charge is $\Omega(|E(G)|)$, and therefore, $|E(G)|=O(n)$.

Let us begin then by assigning charges to the faces of $G^{\prime}$ such that each face $f$ receives a charge of $|f|+v(f)-4$. Summing the total charges over all the faces of $G^{\prime}$, we have

$$
\sum_{f \in F\left(G^{\prime}\right)}(|f|+v(f)-4)=2\left|E\left(G^{\prime}\right)\right|+\sum_{f \in F\left(G^{\prime}\right)} v(f)-4\left|F\left(G^{\prime}\right)\right|=4 n-8,
$$

where the last equality follows from Euler's formula and from the next equalities:

$$
\begin{aligned}
\sum_{f \in F\left(G^{\prime}\right)} v(f) & =\sum_{u \in V(G)} d(u)=\sum_{u \in V\left(G^{\prime}\right)} d(u)-\sum_{u \in X(G)} d(u) \\
& =2\left|E\left(G^{\prime}\right)\right|-4\left(\left|V\left(G^{\prime}\right)\right|-|V(G)|\right) .
\end{aligned}
$$

Our plan is to redistribute the charges such that there will be no faces with a negative charge and every original vertex $v$ will be charged with at least $\frac{d(v)}{36}$ units of charge. Then, the total charge over all the original vertices will be $\frac{2|E(G)|}{36} \leq 4 n-8$, and the theorem will follow. Since a face of size one yields a self-intersecting arc and a face of size two yields an empty lens or two parallel arcs, the only faces with a negative charge are 0 -triangles. We proceed by describing a method to charge these faces. Then, we will show how to charge the original vertices.

Charging 0-triangles Let $t$ be a 0 -triangle, let $e_{1}$ be one of its edges, and let $f_{1}$ be the other face incident to $e_{1}$ (see Fig. 1(a)). It must be that $\left|f_{1}\right|>3$, for otherwise there would be an empty lens. If $v\left(f_{1}\right)>0$ or $\left|f_{1}\right|>4$, we move $\frac{1}{3}$ units of charge from $f_{1}$ to $t$ and say that $f_{1}$ contributed $\frac{1}{3}$ units of charge to $t$ through $e_{1}$. Otherwise, $f_{1}$ must be a 0 -quadrilateral. Let $e_{2}$ be the opposite edge to $e_{1}$ in $f_{1}$, and let $f_{2}$ be the other face incident to $e_{2}$. Applying the same arguments as above, we conclude that either $f_{2}$ contributes $\frac{1}{3}$ units of charge to $t$ through $e_{2}$, or $f$ is also a 0 -quadrilateral. In 
Fig. 2 A pentagon contributing to three 0-triangles through non-consecutive edges implies four pairwise crossing arcs

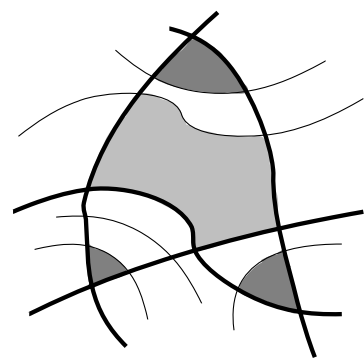

the second case we continue to the next face, that is, to the other face that is incident to the opposite edge to $e_{2}$ in $f_{2}$. However, at some point we must encounter a face that is not a 0 -quadrilateral. Denote by $f_{i}$ this face, by $f_{i-1}$ the face preceding $f_{i}$, and by $e_{i}$ the edge incident to both of these faces. Then $f_{i}$ will contribute $\frac{1}{3}$ units of charge to $t$ through $e_{i}$ (see Figs. 1(b,c)). Note that if $f_{i}$ is a 1-triangle, then it implies that $G$ has an empty lens. Furthermore, both endpoints of $e_{i}$ are crossing points. Thus, $\left|f_{i}\right|>3$.

In a similar way, $t$ obtains $\frac{2}{3}$ units of charge from its other edges. Thus, after redistributing charges this way, the charge of every 0 -triangle is 0 . Note that a face can contribute charges through each of its edges at most once. Therefore, every face $f$ such that $|f|+v(f) \geq 6$ still has a nonnegative charge. Since triangles do not contribute charge, it remains to verify that 1-quadrilaterals and 0-pentagons, which had only one unit of charge to contribute, also have a nonnegative charge. Indeed, a 1-quadrilateral contributes charge to at most two 0-triangles, since the endpoints of an edge through which it contributes charge must be vertices in $X(G)$. A 0 -pentagon can contribute charge to at most three 0 -triangles by the following easy observation.

Observation 2.1 A 0-pentagon contributes charge to at most three 0-triangles. Moreover, if it contributes charge to three 0-triangles, then it must be done through consecutive edges.

Proof One can easily check that a contribution to three 0-triangles through nonconsecutive edges implies four pairwise crossing arcs (see Fig. 2). In case a 0-pentagon contributes charge to more than three 0-triangles, then there must be three nonconsecutive edges through which it contributes charge.

We charge an original vertex $v$ by charging each of the $d(v)$ wedges incident to $v$. Draw a small circle $C$ around $v$ such that $v$ is the only vertex of $G^{\prime}$ in the region bounded by $C$. Then the crossing points of $C$ with the arcs incident to $v$ define a clockwise order of the arcs that are incident to $v$.

Definition 2.2 (Wedge) A wedge is a triplet $w=\left(v, a_{l}, a_{r}\right)$ such that: $v \in V(G) ; a_{l}$ and $a_{r}$ are arcs incident to $v$; and $a_{l}$ immediately follows $a_{r}$ in a clockwise order of the arcs that are incident to $v$.

We say that a face $f \in F\left(G^{\prime}\right)$ is the first face of a wedge $w=\left(v, a_{l}, a_{r}\right)$ if there are points $p_{l} \in a_{l}$ and $p_{r} \in a_{r}$ such that $\left.\left.a_{l}\right|_{v, p_{l}} \cup a_{r}\right|_{v, p_{r}}$ is on the boundary of $f$. 


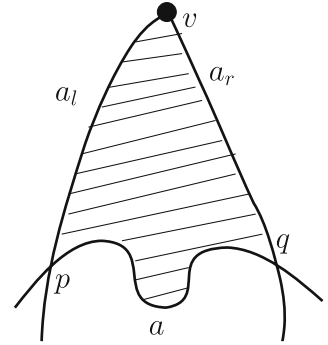

(a) $\left.w\right|_{a, p, q}$

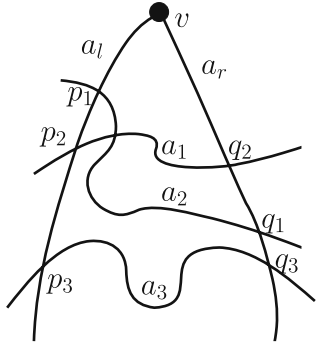

(b) $\Delta$-crossings of a wedge. $\left(a_{3}, p_{3}, q_{3}\right)$ is uncut and is farther than $\left(a_{1}, p_{1}, q_{1}\right) \quad$ and $\left(a_{2}, p_{2}, q_{2}\right)$.

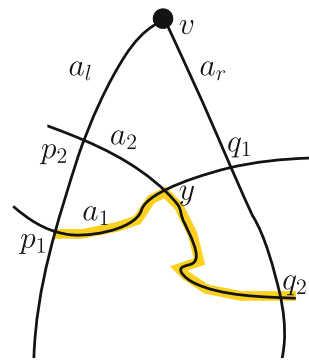

(c) an $X$-crossing of a wedge. The visible part of the $X$ crossing is marked.

Fig. 3 Crossing patterns of a wedge

Charging Wedges After the previous step, the faces with a zero charge, apart from 0 -triangles, 1-triangles, and 0-quadrilateral, are 0-pentagons that contributed charge to three 0-triangles, and 0-hexagons that contributed charge to six 0-triangles. We call such faces bad faces. Faces that have a positive charge are called good faces. It is easy to verify that a good face has at least $1 / 3$ units of charge. Our goal now is to find some extra charge for each wedge. This extra charge will be found next to a farthest uncut $\Delta$-crossing or $X$-crossing of the wedge. At this point, few new definitions are needed.

Let $w=\left(v, a_{l}, a_{r}\right)$ be a wedge, let $a$ be an arc, and let $p$ and $q$ be crossing points of $a$ with $a_{l}$ and $a_{r}$, respectively, such that $\left.a\right|_{p, q}$ does not cross $a_{l}$ or $a_{r}$ in any other point. We denote by $\left.w\right|_{a, p, q}$ the bounded and closed region whose boundary is the closed curve composed of $\left.a_{l}\right|_{v, p},\left.a\right|_{p, q}$, and $\left.a_{r}\right|_{q, v}$ (see Fig. 3(a)).

Definition 2.3 $\Delta$-crossing Let $w=\left(v, a_{l}, a_{r}\right)$ be a wedge. A $\Delta$-crossing of $w$ is a triplet $\delta=(a, p, q)$ such that $a$ is an arc crossing $a_{l}$ at $p$ and $a_{r}$ at $q$ such that: (1) $\left.a\right|_{p, q}$ does not intersect $a_{l}$ or $a_{r}$; and (2) there are no original vertices in the interior of $\left.w\right|_{a, p, q}$. We say that $\delta$ is an uncut $\Delta$-crossing of $w$ if $\left.a\right|_{p, q}$ is an edge of $G^{\prime}$. For illustrations, refer to Fig. 3(b).

Let $\delta=(a, p, q)$ be a $\Delta$-crossing of a wedge $w=\left(v, a_{l}, a_{r}\right)$. We use the notation $\left.w\right|_{\delta}$ as an abbreviation for $\left.w\right|_{a, p, q}$. Given another $\Delta$-crossing of $w, \delta^{\prime}=\left(a^{\prime}, p^{\prime}, q^{\prime}\right)$, we say that $\delta$ is farther than $\delta^{\prime}$ if $\left.p^{\prime} \in a_{l}\right|_{v, p}$ and $\left.q^{\prime} \in a_{r}\right|_{v, q}$. Clearly, not every two $\Delta$-crossings of a wedge are comparable, but uncut $\Delta$-crossings are.

Definition 2.4 ( $X$-crossing) Let $w$ be a wedge, and let $\delta_{1}=\left(a_{1}, p_{1}, q_{1}\right)$ and $\delta_{2}=$ $\left(a_{2}, p_{2}, q_{2}\right)$ be two $\Delta$-crossings of $w$. Then $x=\left(\delta_{1}, \delta_{2}\right)$ is an $X$-crossing of $w$ if $\left.a_{1}\right|_{p_{1}, q_{1}}$ and $\left.a_{2}\right|_{p_{2}, q_{2}}$ intersect exactly once. We use the notation $\left.w\right|_{x}$ to denote the region $\left.\left.w\right|_{\delta_{1}} \cup w\right|_{\delta_{2}}$. 
Fig. 4 An illustration for the proof of Observation 2.6

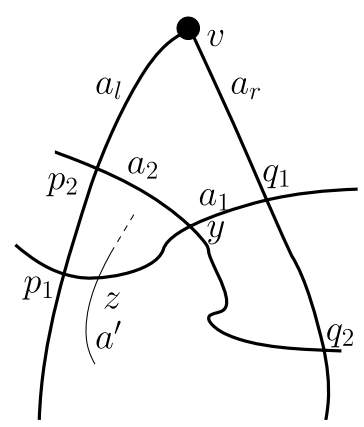

Let $x$ and $x^{\prime}$ be two $X$-crossings of a wedge $w$. We say that $x$ is farther than $x^{\prime}$ if one $\Delta$-crossing of $x$ is farther than one $\Delta$-crossing of $x^{\prime}$ and the other $\Delta$-crossing of $x^{\prime}$ is not farther than the other $\Delta$-crossing of $x$ (note that the last two $\Delta$-crossings might be identical). In a similar way, we say that an uncut $\Delta$-crossing is farther (resp., closer) than an $X$-crossing of the same wedge if it is farther (resp., closer) than both $\Delta$-crossings of the $X$-crossing.

Definition 2.5 (Visible part of an $X$-crossing) Let $x=\left(\delta_{1}=\left(a_{1}, p_{1}, q_{1}\right), \delta_{2}=\right.$ $\left.\left(a_{2}, p_{2}, q_{2}\right)\right)$ be an $X$-crossing of a wedge $w=\left(v, a_{l}, a_{r}\right)$. Then the part of the boundary of $\left.w\right|_{x}$ that is composed of segments of the arcs $a_{1}$ and $a_{2}$ is called the visible part of $\left.w\right|_{x}$ and is denoted by $\operatorname{Vis}(x)$ when it is clear to which wedge we refer. We denote by $\operatorname{Vis}\left(x, a_{l}\right)$ (resp., $\left.\operatorname{Vis}\left(x, a_{r}\right)\right)$ the segment of $\operatorname{Vis}(x)$ whose one endpoint lies on $a_{l}$ (resp., $a_{r}$ ) and whose other endpoint is the intersection point of $\left.a_{1}\right|_{p_{1}, q_{1}}$ and $\left.a_{2}\right|_{p_{2}, q_{2}}$. See Fig. 3(c) for an example.

The next observation will be useful later.

Observation 2.6 Suppose that $x=\left(\delta_{1}=\left(a_{1}, p_{1}, q_{1}\right), \delta_{2}=\left(a_{2}, p_{2}, q_{2}\right)\right)$ is an $X$ crossing of a wedge $w=\left(v, a_{l}, a_{r}\right)$ such that $\operatorname{Vis}\left(x, a_{l}\right) \subset a_{1}$. Then an arc $a^{\prime}$ that crosses $\operatorname{Vis}\left(x, a_{l}\right)$ (resp., $\left.\operatorname{Vis}\left(x, a_{r}\right)\right)$ must cross $a_{l}$ (resp., $\left.a_{r}\right)$ and must not cross $a_{2}$ (resp., $\left.a_{1}\right)$.

Proof Let $y$ be the crossing point of $\left.a_{1}\right|_{p_{1}, q_{1}}$ and $\left.a_{2}\right|_{p_{2}, q_{2}}$, and let $z$ be the crossing point of $a^{\prime}$ and $\left.a_{1}\right|_{p_{1}, y}$ (see Fig. 4). Since the interior of $\left.w\right|_{x}$ contains no original vertex, $a^{\prime}$ must cross the boundary of $\left.w\right|_{x}$ at least one more time. If it crosses $\left.a_{1}\right|_{p_{1}, q_{1}}$ at any point other than $z$, we have an empty lens. Therefore, $a^{\prime}$ cannot cross $\operatorname{Vis}(x)$ at any point but $z$. Otherwise, if $a^{\prime}$ crosses $\left.a_{r}\right|_{v, q_{2}}$, then it must also cross $\left.a_{2}\right|_{p_{2}, y}$. This implies four pairwise crossing arcs: $a^{\prime}, a_{1}, a_{2}$, and $a_{r}$. Thus $a^{\prime}$ must cross $a_{l}$. Moreover, it must not cross $a_{2}$ since this also yields four pairwise crossing edges. The proof for an arc crossing $\operatorname{Vis}\left(x, a_{r}\right)$ is similar and is thus omitted.

Rules for Charging a Wedge We are now ready to define the charging rules for wedges. Given a wedge $w=\left(v, a_{l}, a_{r}\right)$, we look for an uncut $\Delta$-crossing or $X$ crossing of $w$ such that there is no uncut $\Delta$-crossing or $X$-crossing farther than it and continue as follows: 
Fig. 5 A bad pentagon next to an uncut $\Delta$-crossing implies a farther $X$-crossing

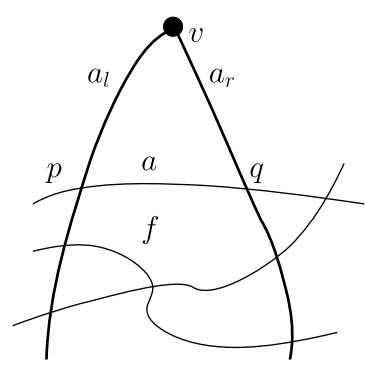

1. Suppose that there is no such uncut $\Delta$-crossing or $X$-crossing. Then the first face of $w$ is not a 1-triangle. Thus, its charge is at least $\frac{1}{3}$ units, from which we use $\frac{1}{36}$ units to charge $w$.

2. Suppose that there is such an uncut $\Delta$-crossing $\delta=(a, p, q)$. Let $f$ be the face incident to $\left.a\right|_{p, q}$ outside $\left.w\right|_{\delta}$. $f$ is not a triangle as this would yield an empty lens or parallel arcs. It also cannot be a 0 -quadrilateral since this would imply an uncut $\Delta$-crossing farther than $\delta$. If $f$ is a bad pentagon, then it follows from Observation 2.1 that there is an $X$-crossing farther than $\delta$ (see Fig. 5). $f$ cannot be a bad hexagon, since this would imply that it has contributed charge to a 0 -triangle through $\left.a\right|_{p, q}$. Therefore, $f$ must be a good face that will contribute $\frac{1}{36}$ units of charge to $w$ through $\left.a\right|_{p, q}$.

3. Suppose that there is such an $X$-crossing $x=\left(\delta_{1}=\left(a_{1}, p_{1}, q_{1}\right), \delta_{2}=\left(a_{2}, p_{2}, q_{2}\right)\right)$. Denote by $y$ the intersection point of $\left.a_{1}\right|_{p_{1}, q_{1}}$ and $\left.a_{2}\right|_{p_{2}, q_{2}}$, and assume w.l.o.g. that $\operatorname{Vis}\left(x, a_{l}\right)=\left.a_{1}\right|_{p_{1}, y}$. Let $f_{1}$ be the face that is incident to $y$ and outside $\left.w\right|_{x}$ (see Fig. 6). Let us analyze the possible subcases.

(a) $f_{1}$ is a good face. Let $e_{i}$ be the edge of $f_{i}$ that is contained in $\operatorname{Vis}\left(x, a_{l}\right)$. Then $f_{i}$ contributes $\frac{1}{36}$ units of charge to $w$ through $e_{i}$.

(b) $f_{1}$ is a 0 -triangle. Let $a_{3}$ be the third arc incident to $f_{1}$ (see Fig. 6(a)). It follows from Observation 2.6 that $a_{3}$ must cross $a_{l}$, thus $a_{l}, a_{1}, a_{2}$, and $a_{3}$ are pairwise crossing. Hence, this subcase is impossible.

(c) If $f_{1}$ is a bad pentagon, then we consider the possible cases according to which none, one, or both of $\left.a_{1}\right|_{p_{1}, y}$ and $\left.a_{2}\right|_{y, q_{2}}$ are edges of $G^{\prime}$. In case both of them are edges (see Fig. 6(b)), then there is an uncut $\Delta$-crossing of $w$ that is farther than $x$. In case one of them, say $\left.a_{2}\right|_{y, q_{2}}$, is an edge (see Fig. 6(c)), then there is an $X$-crossing farther than $x$. If none of them is an edge (see Fig. 6(d)), then there must be four pairwise crossing arcs. Therefore $f_{1}$ cannot be a bad pentagon.

(d) By arguments similar to the previous case it follows that if $f_{1}$ is a bad hexagon, then there must be four pairwise crossing arcs, or an $X$-crossing of $w$ farther than $x$.

(e) $f_{1}$ is a 0 -quadrilateral. Let $f_{2}$ be the face outside of $\left.w\right|_{x}$ that shares an edge with $f_{1}$ and is incident to $\operatorname{Vis}\left(x, a_{l}\right)$ (see Fig. 6(e)). If there is no such face, or there is no face outside $\left.w\right|_{x}$ that shares an edge with $f_{1}$ and is incident to $\operatorname{Vis}\left(x, a_{r}\right)$, then there is an $X$-crossing farther than $x$. Examining $f_{2}$, one can see by inspection that it cannot be a 0-triangle, as this implies four pairwise crossing arcs. If $f_{2}$ is a bad pentagon (see Fig. 6(f, g)) or a bad hexagon, 


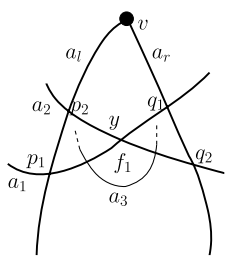

(a) $f_{1}$ is a 0 triangle

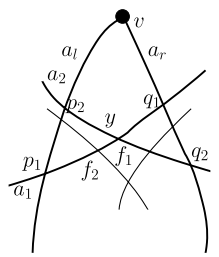

(e) $f_{2}$

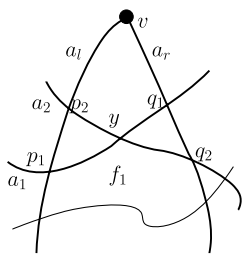

(b) $f_{1}$ is a bad pentagon such that $\left.\quad a_{1}\right|_{p_{1}, y}$ and $\left.a_{2}\right|_{y, q_{2}}$ are edges.

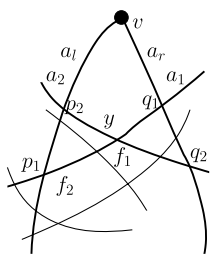

(f) $f_{2}$ is a bad pentagon

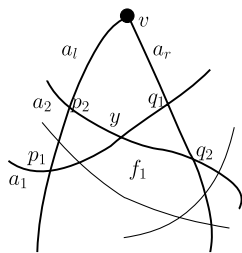

(c) $f_{1}$ is a bad pentagon such that $\left.a_{2}\right|_{y, q_{2}}$ is an edge and $\left.a_{1}\right|_{p_{1}, y}$ is not.

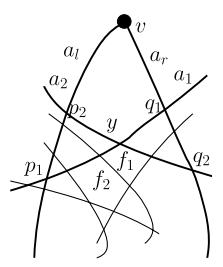

(g) $f_{2}$ is a bad pentagon

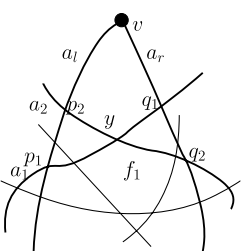

(d) $f_{1}$ is a bad pentagon such that $\left.a_{1}\right|_{p_{1}, y}$ and $\left.a_{2}\right|_{y, q_{2}}$ are not edges.

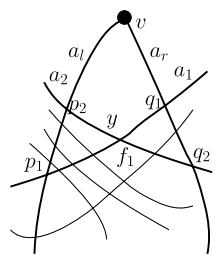

(h) All the
faces along
Vis $\left(x, a_{l}\right)$ are
0-quadrilaterals

Fig. 6 Obtaining a charge near a farthest $X$-crossing

then again there must be four pairwise crossing arcs or an $X$-crossing farther than $x$. Thus, either $f_{2}$ is a good face or it is a 0 -quadrilateral.

In the second case, we examine the "next" face, that is, the face $f_{3} \neq f_{1}$ such that $f_{3}$ is outside $\left.w\right|_{x}$, shares an edge with $f_{2}$, and is incident to $\operatorname{Vis}\left(x, a_{l}\right)$. If there is no such face, then we have an $X$-crossing farther than $x$. Otherwise, we can apply the same arguments we used for $f_{2}$ on $f_{3}$, proceed to the next face if $f_{3}$ is not a good face, and so on. At some point we must encounter a good face, for otherwise we have an $X$-crossing farther than $x$ (see Fig. 6(h)). Let $f_{i}$ be the first good face we encounter along $\operatorname{Vis}\left(x, a_{l}\right)$, and let $e_{i}$ be the edge of $f_{i}$ that is contained in $\operatorname{Vis}\left(x, a_{l}\right)$. Then $f_{i}$ contributes $\frac{1}{36}$ units of charge to $w$ through $e_{i}$.

Analyzing the Final Charges Next, we prove that after applying the above charging rules there are no faces with a negative charge. For that, we need to show that a face cannot contribute charge to "too many" wedges. Apart from Rule 3(e), whenever a face contributes charge to a wedge, the edge through which the contribution is made lies on one arc of the wedge. Therefore, it is easy to see that, following those rules, a face contributes a charge that is proportional to its size. We will also show that Rule 3(a) cannot be applied more than once for a certain edge of a certain face. For this, we introduce the following definition. 


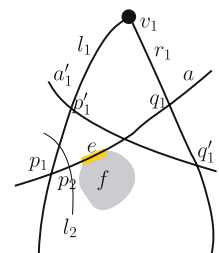

(a) $l_{2}$ must cross $l_{1}$.

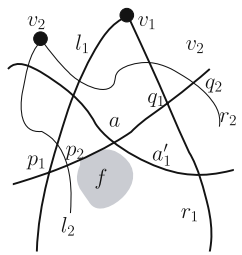

(e) $a_{1}^{\prime}$ crosses $l_{2}$.

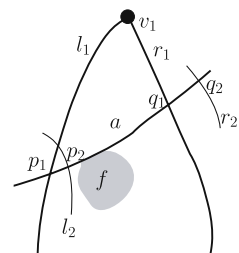

(b) $q_{1}$ precedes $q_{2}$

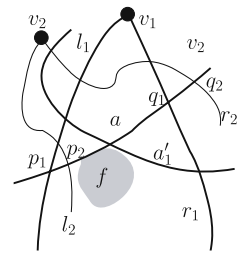

(f) $a_{1}^{\prime}$ crosses $r_{2}$.

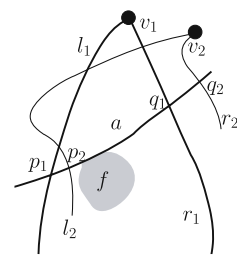

(c) $l_{1}$ and $l_{2}$ form an empty lens.

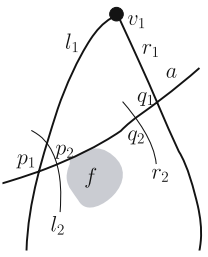

(g) $q_{2}$ precedes $q_{1}$

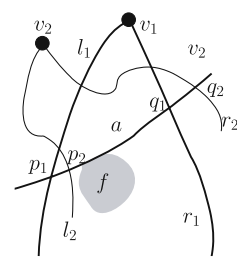

(d) $r_{2}$ crosses $l_{1}$ and $r_{1}$.

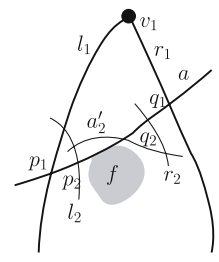

(h) $a_{2}^{\prime}$ must cross $l_{1}$.

Fig. 7 Illustrations for the proof of Observation 2.8

Definition 2.7 (Possible $X$-contributor) For a (good) face $f$ and one of its edges $e$, we say that $f$ is a possible $X$-contributor to a wedge $w=\left(v, a_{l}, a_{r}\right)$ through $e$ if there is an $X$-crossing of $w, x$, such that $f$ is outside $\left.w\right|_{x}$ and $e \subset \operatorname{Vis}\left(x, a_{l}\right)$.

Observation 2.8 Let $f$ be a face, and let e be one of its edges. Then $f$ is a possible $X$-contributor through e to at most one wedge.

Proof Suppose that there is a face $f$ that is a possible $X$-contributor through one of its edges, $e$, to two wedges, $w_{1}=\left(v_{1}, l_{1}, r_{1}\right)$ and $w_{2}=\left(v_{2}, l_{2}, r_{2}\right)$. Let $a$ be the arc containing $e$; then there are four points, $p_{1}, q_{1}, p_{2}, q_{2}$, such that $\delta_{1}=\left(a, p_{1}, q_{1}\right)$ is a $\Delta$-crossing of $w_{1}$ and $\delta_{2}=\left(a, p_{2}, q_{2}\right)$ is a $\Delta$-crossing of $w_{2}$. Denote by $x_{1}=$ $\left(\left(a, p_{1}, q_{1}\right),\left(a_{1}^{\prime}, p_{1}^{\prime}, q_{1}^{\prime}\right)\right)$ the $X$-crossing of $w_{1}$ such that $e \subset \operatorname{Vis}\left(x_{1}, l_{1}\right)$, and by $x_{2}=$ $\left(\left(a, p_{2}, q_{2}\right),\left(a_{2}^{\prime}, p_{2}^{\prime}, q_{2}^{\prime}\right)\right)$ the $X$-crossing of $w_{2}$ such that $e \subset \operatorname{Vis}\left(x_{2}, l_{2}\right)$. Suppose that we sort $p_{1}, q_{1}, p_{2}, q_{2}$ by the order in which they appear when traversing $a$ from one of its endpoints to the other, so that when traversing $e$ the face $f$ is to our right. Then, $p_{i}$ must precede $q_{i}$ for $i=1,2$, since $f$ is outside of $\left.w_{i}\right|_{x_{i}}$. Assume, w.l.o.g., that $p_{1}$ precedes $p_{2}$. It follows from Observation 2.6 that $l_{2}$ crosses $l_{1}$ (see Fig. 7(a)). Since $\left.\left.e \subset a\right|_{p_{1}, q_{1}} \cap a\right|_{p_{2}, q_{2}}$, the order of the four points is either $p_{1}, p_{2}, q_{1}, q_{2}$ or $p_{1}, p_{2}, q_{2}, q_{1}$. Let us consider these cases:

Case 1: Suppose that $q_{1}$ precedes $q_{2}$ (see Fig. 7(b)). Since $\left.w_{1}\right|_{x_{1}}$ and $\left.w_{2}\right|_{x_{2}}$ do not contain any original vertex, either (1) $l_{2}$ must cross $l_{1}$ (one more time) and $r_{1}$ (see Fig. 7(c)) or (2) $r_{2}$ must cross $l_{1}$ and $r_{1}$ (see Fig. 7(d)). The first case yields an empty lens. In the second case, note that $a_{1}^{\prime}$ must cross either $l_{2}$ (see Fig. 7(e)) or $r_{2}$ (see Fig. 7(f)), yielding four pairwise crossing arcs. 
Case 2: Suppose that $q_{2}$ precedes $q_{1}$ (see Fig. $7(\mathrm{~g})$ ). Then the arc $a_{2}^{\prime}$ must cross $a$ twice, creating an empty lens, or cross $l_{1}$, yielding four pairwise crossing arcs (see Fig. 7(h)).

Since all the cases imply forbidden configurations (an empty lens or four pairwise crossing arcs), $f$ cannot be a possible $X$-contributor through $e$ to more than one wedge.

Lemma 2.9 The final charge of every face is nonnegative.

Proof Let $e$ be an edge of a face $f$. Recall that $f$ contributes at most $1 / 3$ units of charge through $e$ when 0 -triangles are being charged. When charging the wedges, Rules 2 and 3 can be applied on $f$ and $e$ at most once each. Note that if Rule 1 is applied on $f$ to charge a wedge with a vertex $v$, then $f$ does not contribute charge through its two edges that are incident to $v$ (the endpoints of an edge through which charge is contributed are always in $X(G))$. Thus, $f$ contributes at most $|f|-4-$ $|f|\left(\frac{1}{3}+\frac{1}{36}+\frac{1}{36}\right)$ units of charge. Therefore, if $|f| \geq 7$, its final charge is nonnegative.

Assume that $|f| \leq 6$. If $f$ is a bad face, then it does not contribute any charge to a wedge, and so its final charge is zero. If $f$ is a good face, then recall that its charge after the 0 -triangles were charge is at least $1 / 3$. When the wedges are being charged, $f$ contributes at most $\frac{2|f|}{36}$ units of charge, thus it remains with a nonnegative charge.

Summing up the charges over all the wedges, we have $\frac{2|E(G)|}{36} \leq 4 n-8$; hence $|E(G)| \leq 72(n-2)$.

\section{Discussion}

We have shown that the maximum number of arcs in a topological graph on $n$ vertices with no four pairwise crossing arcs is $O(n)$. The constant in Theorem 1 is certainly not optimal. One can reduce it by noticing that it is impossible that all the faces incident to a vertex of $G$ are 1-triangles (as done in [1]), or by further analyzing the ways a small-sized face contributes charge. The bound we found, combined with the analysis in [2] and [4], yields the following corollaries.

Corollary 3.1 For any fixed integer $k>4$, a simple topological graph on $n$ vertices with no $k$ pairwise crossing arcs has $O\left(n \log ^{2 k-8} n\right)$ arcs.

Corollary 3.2 For any fixed integer $k>4$, a topological graph on $n$ vertices with no $k$ pairwise crossing arcs has $O\left(n \log ^{4 k-16} n\right)$ arcs.

This improves the previous bounds by a factor of $\Theta\left(\log ^{2} n\right)$ and $\Theta\left(\log ^{4} n\right)$, respectively. However, the conjecture that $f_{k}(n)=O(n)$ for any fixed $k>4$ remains open. It might be possible to settle this conjecture for $k=5$ using our method, but it seems that for greater values, one should come up with new ideas. 
Acknowledgements I am grateful to Rom Pinchasi for introducing this problem to me, for many helpful suggestions and discussions concerning it, and for his comments on a draft of this paper. I also wish to extend my gratitude to Gábor Tardos for his enlightening observations on the addressed problem and for significantly simplifying a preliminary proof of Theorem 1. I thank anonymous referees for carefully reading the paper and pointing out an inaccuracy in an earlier version of the paper.

\section{References}

1. Ackerman, E., Tardos, G.: On the maximum number of edges in quasi-planar graphs. J. Comb. Theory, Ser. A 114(3), 563-571 (2007)

2. Agarwal, P.K., Aronov, B., Pach, J., Pollack, R., Sharir, M.: Quasi-planar graphs have a linear number of edges. Combinatorica 17(1), 1-9 (1997)

3. Pach, J.: Notes on geometric graph theory. In: Goodman, J.E., Pollack, R., Steiger, W. (eds.) Discrete and Computational Geometry: Papers from DIMACS special year. DIMACS Series, vol. 6, pp. 273285. AMS, Providence (1991)

4. Pach, J., Radoičić, R., Tóth, G.: Relaxing planarity for topological graphs. In: Akiyama, J., Kano, M. (eds.) JCDCG. Lecture Notes in Computer Science, vol. 2866, pp. 221-232. Springer, New York (2002)

5. Valtr, P.: Graph drawings with no $k$ pairwise crossing edges. In: Battista, G.D. (ed.) Graph Drawing. Lecture Notes in Computer Science, vol. 1353, pp. 205-218. Springer, New York (1997) 